\title{
Absorption as a selective mechanism in surface plasmon resonance fiber optic sensors
}

\author{
Óscar Esteban \\ Departamento de Electrónica, Universidad de Alcalá, Escuela Politécnica, 28871 Alcalá de Henares, \\ Madrid (Spain) \\ Agustín González-Cano and Natalia Díaz-Herrera \\ Departamento de Óptica, Escuela Universitaria de Óptica, Universidad Complutense de Madrid, \\ Arcos de Jalón, s/n, 28037 Madrid (Spain) \\ María-Cruz Navarrete \\ Departamento de Óptica, Facultad de CC, Físicas, Universidad Complutense de Madrid, Ciudad Universitaria, s/n, \\ 28040 Madrid (Spain)
}

Received May 5, 2006; revised August 16, 2006; accepted August 23, 2006; posted August 28, 2006 (Doc. ID 70659); published October 11, 2006

\begin{abstract}
A new concept of surface plasmon resonance fiber optic sensor is presented. By tuning the plasmon resonance to a wavelength for which the outer medium is absorptive, a significant variation of the spectral transmittance of the device is produced as a function of the concentration of the analyte. With this mechanism, selectivity can be achieved without the need of any functionalization of the surfaces or the use of recognizing elements, which is a very interesting feature for any kind of chemical sensor or biosensor. Doubly deposited uniform-waist tapered fibers are well suited for the development of these new sensors. Multiple surface plasmon resonance, obtainable in those structures, can be used for the development of microspectrometers based on this principle. (C) 2006 Optical Society of America

OCIS codes: 240.6680, 060.2370.
\end{abstract}

Surface plasmon resonance (SPR) has become a standard technique for a huge variety of chemical sensors or biosensors. ${ }^{1}$ Plasmon excitation strongly depends on the refractive index of the surrounding medium, and, in that sense, all these sensors can be considered refractometers. To make the response of the devices selective to a specific analyte, recognizing elements must be added to the plasmon supporting structure. This requires a functionalization of the surfaces and a careful selection of the materials. The idea is to take advantage of the locality of the plasmon resonance and to ensure that the variation in the refractive index detected on the sensor is directly produced by the presence of the selected analyte.

In general, the media considered in SPR spectroscopy are dielectric. Absorption has been taken into account as a possibility of sensitivity enhancement, but not clearly as the basis of a new recognizing technique. ${ }^{2-4}$ Chemical sensors and biosensors usually are based on attenuated total reflection configurations and therefore employ angular interrogation. For this reason, it is not easy to relate the absorptive spectrum of the outer medium, if it exists, with the obtained output signal. At the same time, the theoretical analyses become somewhat obscure, since spectral interrogation is not considered and the concept of matching of the wavelength associated to the plasmon resonance and one of the wavelengths of absorption for the outer medium is not clearly stated. Sometimes the phenomenon is misinterpreted, in our opinion, since the contributions of the imaginary and real parts of the refractive index of the medium are considered as clearly separable and responsible for different variations of the obtained reflectance curves.

Spectral interrogation is a usual procedure with fiber optic sensors. When it is used, plasmons reveal themselves as well-defined dips in the spectral transmittance curves, and we can measure the wavelengths associated with the plasmon resonances and the displacements produced in the positions of these wavelengths by the changes in the refractive index of the outer medium. An evanescent field is commonly used to produce SPR fiber optic sensors, and, among the different techniques proposed, tapered optical fibers have proved to be a very good option. Asymmetric depositions on uniform-waist tapers produce multiple plasmon resonances, and the positions of these resonances can be easily tuned by using double depositions (metal-dielectric), as shown by the authors. ${ }^{5}$

We present here a new concept of SPR fiber optic sensor that is selective to specific analytes without the need of adding any recognizing elements. The idea is to tune the position(s) of the plasmon resonance(s) to wavelength(s) for which the outer medium is absorptive. In those cases, the existence of a nonzero imaginary part of the refractive index is always associated with a strong variation of the real part of the index. Since SPR is critically dependent on the variation of that parameter, it is expected that the presence of the absorptive medium induces a change in the conditions for the plasmon resonance, due not only to the imaginary part but also to the real part. These changes will be clearly observable in the spectral transmittance curve of the system. By using spectral interrogation, the behavior of the sensor is 
easily interpretable and the theoretical models involved become simpler.

To evaluate the potential of the concept, we have considered a doubly deposited (silver and praseodymium oxide) uniform-waist tapered fiber with the following parameters: waist length, $7 \mathrm{~mm}$; waist diameter, $37 \mu \mathrm{m}$; Ag thickness, $25 \mathrm{~nm} ; \mathrm{Pr}_{2} \mathrm{O}_{3}$ thickness, $10 \mathrm{~nm}$. We have calculated the spectral transmittance curves in terms of the refractive index of the surrounding medium by using the theoretical model proposed by Villatoro et al. ${ }^{6}$ modified by us to introduce a matricial formalism. This formalism directly uses boundary conditions for the fields to obtain the reflection and transmission coefficients instead of using Fresnel formulas and was previously used by the authors to model fiber optic sensors based on polished fibers. ${ }^{7}$

As a surrounding medium we have considered methanol with variable concentrations of Rhodamine 6G. This substance presents a strong absorption peak in $530 \mathrm{~nm}$, and, as our simulations show, the structure depicted in the last paragraph supports a plasmon resonance in this same wavelength. In Fig. 1, the refractive index of the medium for a concentration of $0.01 \mathrm{M}$ of Rhodamine is shown. ${ }^{8}$ It is clearly seen that the presence of the Rhodamine produces a significant imaginary part for the index, but also a strong variation of the real part in the region of the absorptive wavelength (anomalous dispersion). By introducing the corresponding values of the refractive index in our model, we have calculated the transmittance curves of the structure when the concentration of the dissolved Rhodamine is varied.

In Fig. 2 we can see how the plasmon is clearly defined for the considered wavelength when we have pure methanol as outer medium and how the transmittance corresponding to that wavelength is increased with the presence of Rhodamine. A certain (a)

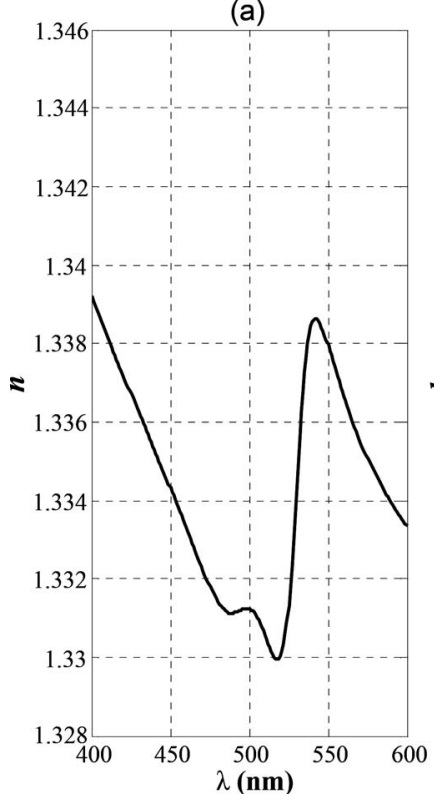

(b)

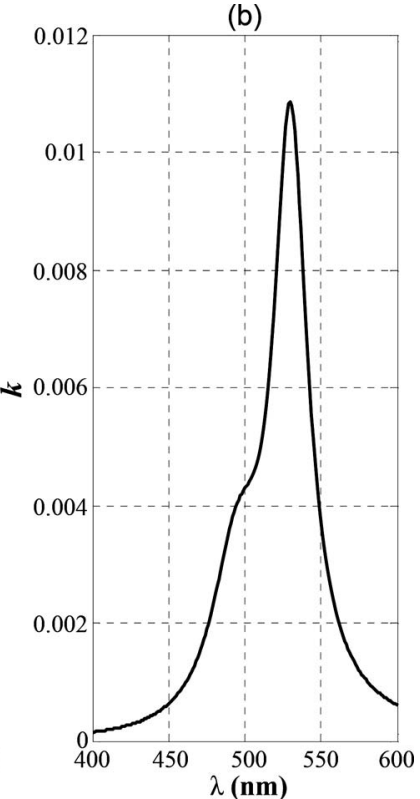

Fig. 1. Refractive index corresponding to a concentration of $0.01 \mathrm{M}$ of Rhodamine $6 \mathrm{G}$ in methanol. (a) and (b) are the real and imaginary parts of the solution, respectively.

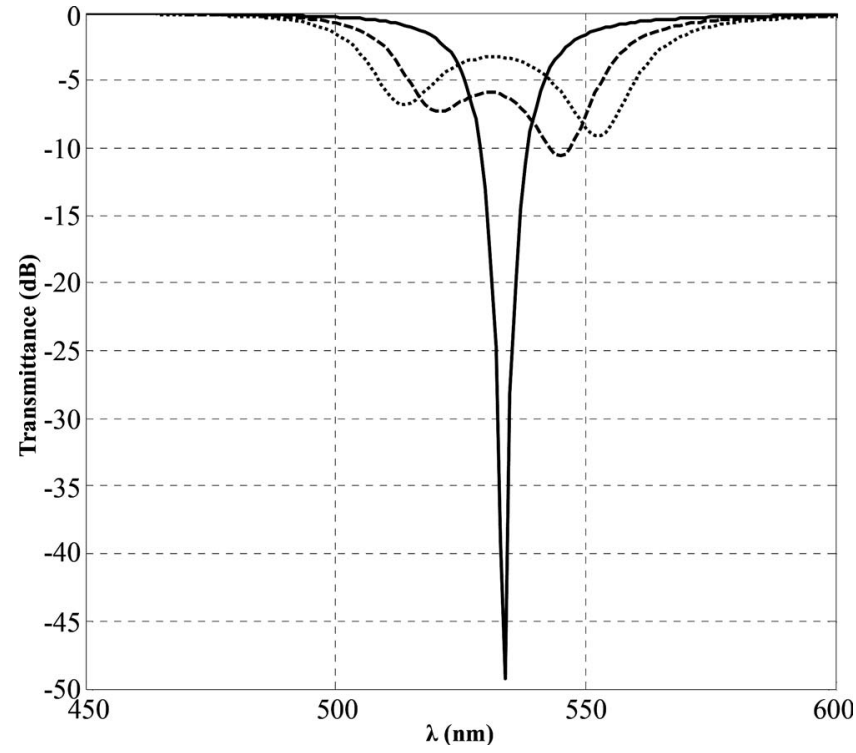

Fig. 2. Effect of the presence of Rhodamine 6G in the plasmon excitation. Solid curve, pure methanol as the outer medium, dashed curve, $0.01 \mathrm{M}$ solution, dotted curve, $0.02 \mathrm{M}$ solution.

"inhibition" of the original plasmon is produced, and two new, less marked minima appear. The increase in the transmittance is directly related to the concentration of Rhodamine. This effect is very easily observable since we have considered spectral interrogation. It is remarkable that the existence of an absorptive medium does not further decrease the transmittance of the system, contrary to what intuition would suggest and to the interpretations of some authors. This is due to the fact that the variation of the real part of the refractive index modifies the conditions for the plasmon resonance. It can be seen that, by measuring the transmittance in a single wavelength, namely, that of the original plasmon, we can determine the concentration of the absorptive species without the need of any treatment of the plasmon supporting structures.

To separate the contributions of the real and imaginary parts of the index we have simulated the effect that substances with only one of these parts would have in the response of the system. The results are shown in Fig. 3. As is well known, a variation of a real index basically produces only a displacement of the dip, without a strong variation of the transmittance. However, since we are dealing with anomalous dispersion, we can see that a local transmittance maximum appears at the absorption wavelength. When only the imaginary part is considered, the plasmon has essentially disappeared. This behavior has not been considered to date, since it is only clearly revealed when spectral interrogation is considered. If the plasmon is not tuned with the absorption wavelength, this behavior is not observed. In this way, we can see that we can achieve specificity and selectivity for these simple structures only by conveniently choosing the materials and the thickness of the layers, since other substances that can be present in the medium would only contribute to the response in the ordinary way. 

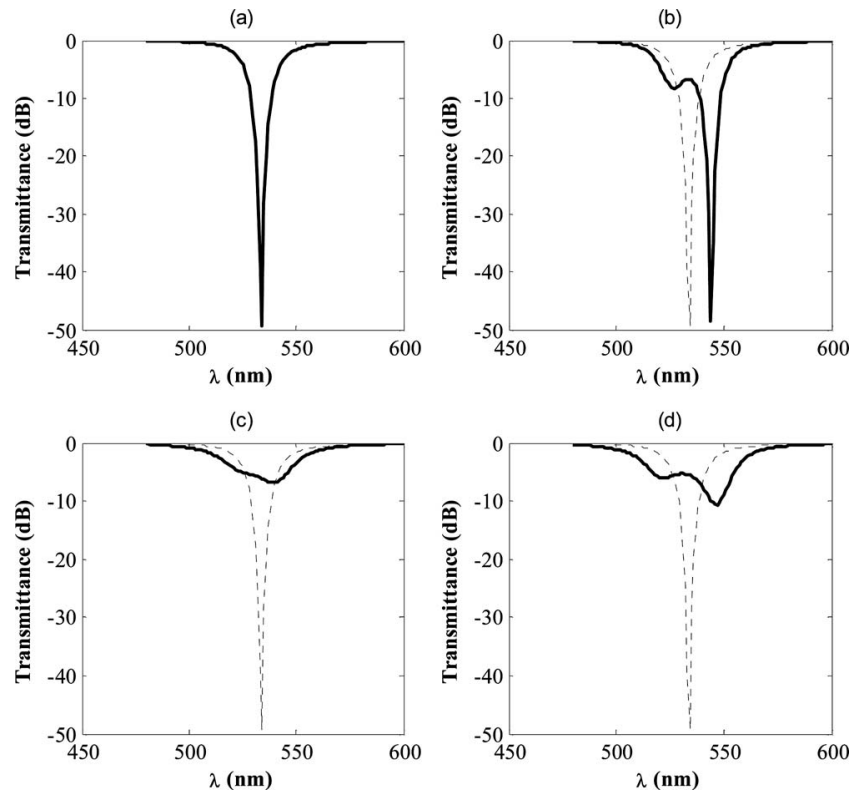

Fig. 3. Effects of the real and imaginary parts of the index shown in Fig. 1 in the transmittance of the device: (a) pure methanol, (b) only the real part, (c) only the imaginary part, (d) both parts.

It is clear that the key point of the technique is the ability to tune the plasmon resonance with the absorption wavelengths. It is well known that the use of a dielectric layer permits shifting of the wavelength of the plasmon resonance as desired. ${ }^{5}$ By using common metals such as $\mathrm{Au}, \mathrm{Ag}$, or $\mathrm{Al}$ and also common dielectrics such as $\mathrm{Al}_{2} \mathrm{O}_{3}, \mathrm{TiO}_{2}$, or $\operatorname{Pr}_{2} \mathrm{O}_{3}$, and selecting the thickness of the layers, we can in principle place the plasmon resonance in any desired wavelength in the visible-near-infrared range with enough accuracy. Of course, we will need to adapt the design of the structure to the target analyte.
This measuring principle can be extended by considering the possibility of simultaneously exciting different plasmons, which can be achieved with asymmetrically deposited tapers. If we tune the plasmon resonances to two or more different characteristic absorptions of the analyte we can improve its identification and reduce the limits of detection. If we tune the resonances to the absorption of two or more different substances, we can simultaneously detect them with the same simple structure. This can be used as the basis of a new concept of SPR fiber optic microspectrometer.

This work has been partially supported by Spanish government project NESTOR (CTM2004-03899/ MAR) and Community of Madrid project FUTURSEN (S-0505/AMB-0374). O. Esteban's e-mail address is oscar@depeca.uah.es.

\section{References}

1. J. Homola, S. S. Yee, and G. Gauglitz, Sens. Actuators B 54, 3 (1999).

2. K. Kurihara and K. Suzuki, Anal. Chem. 74, 696 (2002).

3. A. A. Kolomenskii, P. D. Gershon, and H. A. Schuessler, Appl. Opt. 39, 3314 (2000).

4. A. Hanning, J. Roeraade, J. J. Delrow, and R. C. Jorgenson, Sens. Actuators B 54, 25 (1999).

5. A. González-Cano, F. J. Bueno, Ó. Esteban, N. DíazHerrera, and M. C. Navarrete, Appl. Opt. 44, 519 (2005).

6. J. Villatoro, D. Monzón-Hernández, and E. Mejía, Appl. Opt. 42, 2278 (2003).

7. Ó. Esteban, M. C. Navarrete, A. González-Cano, and E. Bernabeu, Opt. Lasers Eng. 33, 219 (2000).

8. W. Leupacher and A. Penzkofer, Appl. Opt. 23, 1554 (1984) 